\title{
A note on the roots of some special type polynomials
}

\author{
Serpil Halıcl* and Zeynep Akyüz
}

"Correspondence:

shalici@sakarya.edu.tr

Department of Mathematics,

Faculty of Arts and Science, Sakarya

University, Sakarya, 54187, Turkey

\begin{abstract}
In this study, we investigate the polynomials for $n \geq 2$ and positive integers $k$ and a positive real number $a$, with the initial values $G_{0}(x)=-a, G_{1}(x)=x-a$$$
G_{n+2}^{(k)}(x)=x^{k} G_{n+1}^{(k)}(x)+G_{n}^{(k)}(x) .
$$

We give some fundamental properties related to them. Also, we obtain asymptotic results for the roots of polynomials $G_{n}^{(k)}(x)$.
\end{abstract}

MSC: 11B39; $11 \mathrm{~B} 37$

Keywords: Fibonacci polynomials; Binet formula; generating function

\section{Introduction}

The polynomials defined by Catalan, for $n \geq 0$, as follows

$$
F_{n+2}(x)=x F_{n+1}(x)+F_{n}(x) ; \quad F_{1}(x)=1, \quad F_{2}(x)=x
$$

are called Fibonacci polynomials and denoted by $F_{n}(x)$, [1]. The Fibonacci-type polynomials $G_{n}(x), n \geq 0$, are defined by

$$
G_{n+2}(x)=x G_{n+1}(x)+G_{n}(x)
$$

where $G_{0}(x)$ and $G_{1}(x)$ are seed polynomials. There are several studies about the properties of zeros of polynomials $G_{n}(x)$. However, there are no general formulas for zeros of Fibonacci-type polynomials. In [2, 3], the authors studied the limiting behavior of the maximal real roots of polynomials $G_{n}(x)$ with the initial values $G_{0}(x)=-1, G_{1}(x)=x-1$. In [4], the authors generalized Moore's result for these polynomials. They considered the initial values $G_{0}(x)=a, G_{1}(x)=x+b$, where $a$ and $b$ are integer numbers. In [5], the author determined the absolute values of complex zeros of these polynomials. In [6], Ricci studied this problem in the case $a=1$ and $b=1$. In [7], Tewodros investigated the convergence of maximal real roots of different Fibonacci-type polynomials given by the following relation:

$$
G_{n+2}^{(k)}(x)=x^{k} G_{n+1}^{(k)}(x)+G_{n}^{(k)}(x), \quad n \geq 0,
$$

○2013 Halıı and Akyüz; licensee Springer. This is an Open Access article distributed under the terms of the Creative Commons Attribution License (http://creativecommons.org/licenses/by/2.0), which permits unrestricted use, distribution, and reproduction in any medium, provided the original work is properly cited. 
where $k$ is a positive integer number. The initial values of the recursive relation (3) are $G_{0}^{(k)}(x)=-1$ and $G_{1}^{(k)}(x)=x-1$. In this study, firstly, we investigate some fundamental properties of Fibonacci-type polynomials. We give some combinatorial identities related to equation (3). Then, we investigate the limit of maximal real roots of these polynomials. We notice that Tewodros [7] studied a special case $a=1$ of the polynomials we investigate.

2 Some fundamental properties of polynomials $G_{n}^{(k)}(x)$

In this section, we give some fundamental properties of polynomials $G_{n}^{(k)}(x)$, for $n \geq 0$, defined by the recursive formula as follows:

$$
G_{n+2}^{(k)}(x)=x^{k} G_{n+1}^{(k)}(x)+G_{n}^{(k)}(x) ; \quad G_{0}^{(k)}(x)=-a ; \quad G_{1}^{(k)}(x)=x-a .
$$

The characteristic equation for (4) is $t^{2}-x^{k} t-1=0$ and its roots are

$$
\alpha(x)=\frac{x^{k}+\sqrt{x^{2 k}+4}}{2}
$$

and

$$
\beta(x)=\frac{x^{k}-\sqrt{x^{2 k}+4}}{2} .
$$

Note that $\alpha(x) \beta(x)=-1, \alpha(x)+\beta(x)=x^{k}$ and $\alpha(x)-\beta(x)=\sqrt{x^{2 k}+4}$. For relation (4), the Binet formula is

$$
G_{n}^{(k)}(x)=A(x) \alpha^{n}(x)+B(x) \beta^{n}(x)
$$

where

$$
A(x)=\frac{2(x-a)+a x^{k}-a \sqrt{x^{2 k}+4}}{2 \sqrt{x^{2 k}+4}}, \quad B(x)=\frac{-2(x-a)-a x^{k}-a \sqrt{x^{2 k}+4}}{2 \sqrt{x^{2 k}+4}} .
$$

Proposition 2.1 For $n \geq 0$, the generating function for polynomials $G_{n}^{(k)}(x)$ is

$$
H_{r}^{(k)}(x, t)=\sum_{n \geq 0} G_{n+r}^{(k)}(x) t^{n}= \begin{cases}\frac{G_{r}^{(k)}(x)+G_{r-1}^{(k)}(x) t}{1-k^{k} t-t^{2}}, & r=1,2,3, \ldots, \\ \frac{t\left(a x^{k}+x-a\right)}{1-x^{k} t-t^{2}}, & r=0 .\end{cases}
$$

Proof Let $H_{r}^{(k)}(x, t)$ be the generating function for polynomials $G_{n+r}^{(k)}(x)$. So, we write

$$
H_{r}^{(k)}(x, t)=\sum_{n \geq 0} G_{n+r}^{(k)}(x) t^{n}
$$

If we multiply both sides of equation (8) by $x^{k} t$ and $t^{2}$, respectively, then we can get

$$
x^{k} t H_{r}^{(k)}(x, t)=x^{k} G_{r}^{(k)}(x) t^{1}+x^{k} G_{r+1}^{(k)}(x) t^{2}+x^{k} G_{r+2}^{(k)}(x) t^{3}+\cdots+x^{k} G_{r+n-1}^{(k)}(x) t^{n}+\cdots
$$

and

$$
t^{2} H_{r}^{(k)}(x, t)=G_{r}^{(k)}(x) t^{2}+G_{r+1}^{(k)}(x) t^{3}+G_{r+2}^{(k)}(x) t^{4}+\cdots+G_{r+n-2}^{(k)}(x) t^{n}+\cdots .
$$


The last two equations give us the following equation:

$$
\begin{aligned}
H_{r}^{(k)}(x, t)-x^{k} t H_{r}^{(k)}(x, t)-t^{2} H_{r}^{(k)}(x, t)= & G_{r}^{(k)}(x) t^{0}+\left(G_{r+1}^{(k)}(x)-x^{k} G_{r}^{(k)}(x)\right) t \\
& +\left(G_{r+2}^{(k)}(x)-x^{k} G_{r+1}^{(k)}(x)-G_{r}^{(k)}(x)\right) t^{2}+\cdots \\
& +\left(G_{n+r}^{(k)}(x)-x^{k} G_{n+r-1}^{(k)}(x)-G_{n+r-2}^{(k)}(x)\right) t^{n}+\cdots
\end{aligned}
$$

If we use the recurrence relation and simplify it, we write

$$
H_{r}^{(k)}(x, t)-x^{k} t H_{r}^{(k)}(x, t)-t^{2} H_{r}^{(k)}(x, t)=G_{r}^{(k)}(x) t^{0}+\left(G_{r+1}^{(k)}(x)-x^{k} G_{r}^{(k)}(x)\right) t,
$$

i.e.,

$$
H_{r}^{k}(x, t)= \begin{cases}\frac{G_{r}^{(k)}(x)+G_{r-1}^{(k)}(x) t}{1-x^{k} t-t^{2}}, & r=1,2,3, \ldots, \\ \frac{t\left(a x^{k}+x-a\right)}{1-x^{k} t-t^{2}}, & r=0 .\end{cases}
$$

Thus, the proof is completed.

Let us give the well-known formula, which is called the Cassini-like formula, without proof.

Proposition 2.2 (Cassini-like) For $n \geq 0$, we have

$$
G_{n-1}^{(k)}(x) G_{n+1}^{(k)}(x)-\left[G_{n}^{(k)}(x)\right]^{2}=(-1)^{n-1}[A(x) B(x)],
$$

where

$$
A(x)=\frac{2(x-a)+a x^{k}-a \sqrt{x^{2 k}+4}}{2 \sqrt{x^{2 k}+4}}
$$

and

$$
B(x)=\frac{-2(x-a)-a x^{k}-a \sqrt{x^{2 k}+4}}{2 \sqrt{x^{2 k}+4}} .
$$

In the following propositions, we give some sums formulas related to polynomials $G_{n}^{(k)}(x)$.

Proposition 2.3 For $N \geq 0$, we have

$$
H_{0}^{(k)}(x, 1)-H_{N+1}^{(k)}(x, 1)=\sum_{r=0}^{N} G_{r}^{(k)}(x)=\frac{2 a-x-a x^{k}+G_{N+1}^{(k)}(x)+G_{N}^{(k)}(x)}{x^{k}} .
$$

Proof Proof of formula (10) follows now immediately from (7).

Proposition 2.4 For $N \geq 0$, we have the following sum formulas:

$$
\sum_{r=0}^{N} G_{2 r}^{(k)}(x)=\frac{-x^{k+1}-a x^{k}\left(x^{k}-1\right)-G_{2 N}^{(k)}(x)+G_{2 N+2}^{(k)}(x)}{x^{2 k}}
$$


and

$$
\sum_{r=0}^{N} G_{2 r+1}^{(k)}(x)=\frac{a x^{k}-G_{2 N+1}^{(k)}(x)+G_{2 N+3}^{(k)}(x)}{x^{2 k}}
$$

Proof From the Binet formula, we can write

$$
\sum_{r=0}^{N} G_{2 r}^{(k)}(x)=\left(\frac{x-a+a \beta}{\alpha-\beta}\right) \sum_{r=0}^{N} \alpha^{2 r}-\left(\frac{x-a+a \alpha}{\alpha-\beta}\right) \sum_{r=0}^{N} \beta^{2 r},
$$

where $\alpha=\alpha(x), \beta=\beta(x)$. If we substitute the equations

$$
\sum_{r=0}^{N} \alpha^{2 r}=\frac{1-\alpha^{2 N+2}}{1-\alpha^{2}}, \quad \sum_{r=0}^{N} \beta^{2 r}=\frac{1-\beta^{2 N+2}}{1-\beta^{2}}
$$

and

$$
\left(1-\alpha^{2}\right)\left(1-\beta^{2}\right)=-x^{2 k}
$$

into equation (13), then we can get

$$
\sum_{r=0}^{N} G_{2 r}^{(k)}(x)=\left(\frac{x-a+a \beta}{\alpha-\beta}\right) \frac{1-\alpha^{2 N+2}}{1-\alpha^{2}}-\left(\frac{x-a+a \alpha}{\alpha-\beta}\right) \frac{1-\beta^{2 N+2}}{1-\beta^{2}} .
$$

If we rearrange the last equation, then we have

$$
\begin{aligned}
\sum_{r=0}^{N} G_{2 r}^{(k)}(x)= & \frac{-a(\alpha-\beta)+(x-a)\left(\alpha^{2}-\beta^{2}\right)+a\left(\alpha^{3}-\beta^{3}\right)}{\left(1-\alpha^{2}\right)\left(1-\beta^{2}\right)(\alpha-\beta)} \\
& -\frac{\left[(x-a+a \beta) \alpha^{2 N+2}-(x-a+a \alpha) \beta^{2 N+2}\right]}{\left(1-\alpha^{2}\right)\left(1-\beta^{2}\right)(\alpha-\beta)} \\
& +\frac{\alpha^{2} \beta^{2}\left[(x-a+a \beta) \alpha^{2 N}-(x-a+a \alpha) \beta^{2 N}\right]}{\left(1-\alpha^{2}\right)\left(1-\beta^{2}\right)(\alpha-\beta)} .
\end{aligned}
$$

By taking aid of the Binet formula, we can write

$$
\begin{aligned}
\sum_{r=0}^{N} G_{2 r}^{(k)}(x)= & \frac{-a+(x-a)(\alpha+\beta)+a\left(\alpha^{2}+\alpha \beta+\beta^{2}\right)}{\left(1-\alpha^{2}\right)\left(1-\beta^{2}\right)} \\
& +\frac{G_{2 N}^{(k)}(x)}{\left(1-\alpha^{2}\right)\left(1-\beta^{2}\right)}-\frac{G_{2 N+2}^{(k)}(x)}{\left(1-\alpha^{2}\right)\left(1-\beta^{2}\right)}
\end{aligned}
$$

If we substitute $\alpha+\beta=x^{k}, \alpha \beta=-1,\left(1-\alpha^{2}\right)\left(1-\beta^{2}\right)=-x^{2 k}$ into the last equation, we obtain the following equation:

$$
\sum_{r=0}^{N} G_{2 r}^{(k)}(x)=\frac{x^{k+1}-a x^{k}+a x^{2 k}-G_{2 N+2}^{(k)}(x)+G_{2 N}^{(k)}(x)}{-x^{2 k}}
$$

Thus, the proof is completed. Similarly, the second part of the proposition can be seen. 


\section{Asymptotic behaviors of the maximal roots for polynomials $G_{n}^{(k)}(x)$}

In this section, firstly for $k=2$, we investigate the roots of polynomials $G_{n}^{(k)}(x)$. After that, we generalize the obtained results for all positive real numbers $k$. When $k=2$, we write

$$
G_{n+2}^{(2)}(x)=x^{2} G_{n+1}^{(2)}(x)+G_{n}^{(2)}(x)
$$

where

$$
G_{0}^{(2)}(x)=-a, \quad G_{1}^{(2)}(x)=x-a
$$

and $a$ is a positive real number. Now, we can give the following lemma to be used the later.

Lemma 3.1 If $r$ is a maximal root of a function $f$ with positive leading coefficient, then $f(x)>0$ for all $x>r$. Conversely, if $f(x)>0$ for all $x \geq t$, then $r<t$. If $f(s)<0$, then $s<r[2]$.

Lemma 3.2 For $n \geq 2, G_{n}^{(2)}(x)$ has at least one real root on the interval $(a, a+1)$ and $g_{n} \in$ $(a, a+1)$, where $g_{n}$ is the maximal root of polynomial $G_{n}^{(2)}(x)$.

Proof Some of polynomials $G_{n}^{(2)}(x)$ are as follows:

$$
\begin{aligned}
& G_{2}^{(2)}(x)=x^{3}-a x^{2}-a, \\
& G_{3}^{(2)}(x)=x^{5}-a x^{4}-a x^{2}+x-a, \\
& G_{4}^{(2)}(x)=x^{7}-a x^{6}-a x^{4}+2 x^{3}-2 a x^{2}-a,
\end{aligned}
$$

Note that polynomials $G_{n}^{(2)}(x)$ are monic polynomials with degree $n$ and constant term - $a$. If we write for $x=a$, then we have

$$
\begin{aligned}
& G_{1}^{(2)}(a)=0 \\
& G_{2}^{(2)}(a)=-a<0 \\
& G_{3}^{(2)}(a)=-a^{3}=a^{2} G_{2}^{(2)}(a)<0, \\
& G_{4}^{(2)}(a)=-a^{5}-a \leq-a^{5}=a^{2} G_{3}^{(2)}(a)<0,
\end{aligned}
$$

For $k \geq 2$, if we suppose $G_{k}^{(2)}(a) \leq a^{2} G_{k-1}^{(2)}(a)<0$, then by using the recursive relation (14), we get

$$
G_{k+1}^{(2)}(a)=a^{2} G_{k}^{(2)}(a)+G_{k-1}^{(2)}(a)<0 .
$$

Thus, for $x=a$, we get $G_{n}^{(2)}(x)<0$. Similarly, when $x=a+1$, we have $G_{n}^{(2)}(x)>0$. Therefore, $G_{n}^{(2)}(x)$ has at least one real root on the interval $(a, a+1)$, and we write $g_{n} \in(a, a+1)$ for the maximal root of $G_{n}^{(2)}(x)$, which results easily from Lemma 3.1 and the recursive relation for $G_{n}^{(2)}(x)$. 
Let $g_{n}$ denote the maximal root of polynomial $G_{n}^{(2)}(x)$ for every $n \in \mathbb{N}$. Then we can give the following proposition to illustrate the monotonicity of $\left\{g_{2 n-1}\right\}$ and $\left\{g_{2 n}\right\}$.

Proposition 3.3 The sequence $\left\{g_{2 n-1}\right\}$ is a monotonically increasing sequence and the sequence $\left\{g_{2 n}\right\}$ is a monotonically decreasing sequence.

Proof Firstly, we consider polynomials $G_{n}^{(2)}(x)$ with odd indices. By a direct computation, we get $G_{3}^{(2)}(a)=-a^{3}<0, g_{3}>a, a=g_{1}$. Assume that $g_{1}<g_{3}<g_{5}<\cdots<g_{2 k-3}<g_{2 k-1}$. We can write $G_{2 k-3}^{(2)}\left(g_{2 k-1}\right)>0$. Thus, it can be easily seen that

$$
G_{n+k}^{(2)}\left(g_{n}\right)=(-1)^{k+1} G_{n-k}^{(2)}\left(g_{n}\right) .
$$

By using equation (15), we can write

$$
G_{2 k+1}^{(2)}\left(g_{2 k-1}\right)=G_{(2 k-1)+2}^{(2)}\left(g_{2 k-1}\right)=-G_{(2 k-1)-2}^{(2)}\left(g_{2 k-1}\right)=-G_{2 k-3}^{(2)}\left(g_{2 k-1}\right) .
$$

So, from equation (16) we write

$$
G_{2 k+1}^{(2)}\left(g_{2 k-1}\right)<0
$$

Therefore, polynomials $G_{2 k+1}^{(2)}(x)$ must have a root greater than $g_{2 k-1}$. So, we get

$$
g_{2 k+1}>g_{2 k-1} \text {. }
$$

After that we consider polynomials $G_{n}^{(2)}(x)$ with even indices. From the recursive relation (14), we can obtain

$$
G_{2 k+1}^{(2)}\left(g_{2 k-1}\right)=g_{2 k-1}^{2} G_{2 k}^{(2)}\left(g_{2 k-1}\right)+G_{2 k-1}^{(2)}\left(g_{2 k-1}\right) .
$$

Since $G_{2 k-1}^{(2)}\left(g_{2 k-1}\right)=0$, by using Lemma 3.1, we can get $G_{2 k+1}^{(2)}\left(g_{2 k-1}\right)<0$. Thus, we get

$$
g_{2 k-1}<g_{2 k} .
$$

Again, by using Lemma 3.1, we can write

$$
G_{2 k-1}^{(2)}\left(g_{2 k}\right)>0 \text {. }
$$

From the recursive relation (14), we can write

$$
G_{2 k}^{(2)}\left(g_{2 k}\right)=g_{2 k}^{2} G_{2 k-1}^{(2)}\left(g_{2 k}\right)+G_{2 k-2}^{(2)}\left(g_{2 k}\right)
$$

From equation (22), we can get

$$
-g_{2 k}^{2} G_{2 k-1}^{(2)}\left(g_{2 k}\right)=G_{2 k-2}^{(2)}\left(g_{2 k}\right)<0 .
$$

So, we have $g_{2 k}<g_{2 k-2}$. Thus, $\left\{g_{2 n-1}\right\}$ is a monotonically increasing sequence and bounded above by the number $a+1$. Similarly, $\left\{g_{2 n}\right\}$ is a monotonically decreasing sequence and 
bounded below by the number $a$. If we denote the $\lim _{x \rightarrow \infty} g_{2 n-1}$ by $g_{\text {odd }}$ and $\lim _{x \rightarrow \infty} g_{2 n}$ by $g_{\text {even }}$, then we can write $g_{\text {odd }}=g_{\text {even }}$.

Proposition 3.4 For polynomials $G_{2 n-1}^{(2)}(x)$ and $G_{2 n}^{(2)}(x)$, the sequences $\left\{g_{2 n-1}\right\}$ and $\left\{g_{2 n}\right\}$ converge to the following number $\zeta$ :

$$
\zeta=\frac{\sqrt{\left(1-a^{2}\right)^{2}+8 a^{2}}-\left(1-a^{2}\right)}{2 a} .
$$

Proof Using the Binet formula of relation (14), for all $[a, a+1]$, we can see that $\alpha(x) \geq$ $\alpha(a)>1$ and $|\beta(x)|=\frac{1}{\alpha(x)} \leq \frac{1}{\alpha(a)}$. Thus, we get

$$
\lim _{n \rightarrow \infty} \alpha^{n}(x)=+\infty ; \quad \lim _{n \rightarrow \infty} \beta^{n}(x)=0 .
$$

If we write $n=2 k-1$ and $x=g_{2 k-1}$ in equation (5), then we have

$$
A\left(g_{2 k-1}\right) \alpha^{2 k-1}\left(g_{2 k-1}\right)+B\left(g_{2 k-1}\right) \beta^{2 k-1}\left(g_{2 k-1}\right)=0 .
$$

And from equation (25) we write

$$
A\left(g_{2 k-1}\right)=-B\left(g_{2 k-1}\right)\left(\frac{\beta^{2 k-1}\left(g_{2 k-1}\right)}{\alpha^{2 k-1}\left(g_{2 k-1}\right)}\right) .
$$

$A(x)$ and $B(x)$ are continuous on the interval $[a, a+1]$, this implies that $|A(x)|$ and $|B(x)|$ are bounded below and above on $[a, a+1]$. So, since $a \geq 1$, we get

$$
\lim _{k \rightarrow \infty} A\left(g_{2 k-1}\right)=A\left(g_{\text {odd }}\right)=0 .
$$

From Binet formula (5), we have

$$
\lim _{k \rightarrow \infty} g_{2 k-1}=\frac{\sqrt{\left(1-a^{2}\right)^{2}+8 a^{2}}-\left(1-a^{2}\right)}{2 a} .
$$

Also, by the aid of similar discussion, if we take $n=2 k$ and $x=g_{2 k}$, then we find that

$$
\lim _{k \rightarrow \infty} g_{2 k}=\frac{\sqrt{\left(1-a^{2}\right)^{2}+8 a^{2}}-\left(1-a^{2}\right)}{2 a} .
$$

That is,

$$
\zeta=\frac{\sqrt{\left(1-a^{2}\right)^{2}+8 a^{2}}-\left(1-a^{2}\right)}{2 a} .
$$

Notice that if we take $a=1$ in equation (29), then our result coincides with the result of Tewodros [7].

For $\zeta$ numbers in equation (23), from Proposition 3.4 we can deduce the following result. 
Corollary 3.5 For every positive integer a, we have

$$
a<\zeta<a+1
$$

Now, we give a proposition for the maximal real roots of $G_{n}^{(k)}(x)$ without proof.

Proposition 3.6 The maximal real roots of $G_{n}^{(k)}(x)$ provide the following equation:

$$
g-2 a+a g^{k}-a^{2} g^{k-1}=0,
$$

where the numbers $g=g_{n}=g_{n}(k)$ are the maximal real roots of $G_{n}^{(k)}(x)$, that is,

$$
a g_{n}=a^{2}-g_{n}^{2-k}+2 a g_{n}^{1-k}
$$

which implies

$$
\frac{a}{1+a(a+1)^{k-1}}<g_{n}(k)-a<\frac{a}{1+a^{k}}
$$

whenever $k>2$.

$$
\frac{a-1}{a(a+1)}<\frac{2 a-g_{n}(2)}{a g_{n}(2)}=g_{n}(2)-a<\frac{1}{a+1}
$$

and

$$
\lim _{k \rightarrow \infty} g_{n}(k)=a,
$$

whenever $a>1$ for every $n \in \mathbb{N}$.

Proof The proof can be easily seen as being similar to the proof of Proposition 3.4

Competing interests

The authors declare that they have no competing interests.

\section{Authors' contributions}

All authors completed the paper together. All authors read and approved the final manuscript.

\section{Acknowledgements}

The authors are very grateful to the referees for very helpful suggestions and comments about the paper which improved the presentation and its readability.

Received: 18 October 2012 Accepted: 20 September 2013 Published: 07 Nov 2013

\section{References}

1. Koshy, T: Fibonacci and Lucas Numbers with Applications. Wiley, New York (2001)

2. Matyas, F: Bounds for the zeros of Fibonacci-like polynomials. Acta Acad. Paedagog. Agriensis Sect. Mat. 25, 15-20 (1998)

3. Moore, GA: The limit of the golden numbers is 3/2. Fibonacci Q. 32(3), 211-217 (1993)

4. Prodinger, H: The asymptotic behavior of the golden numbers. Fibonacci Q. 34(3), 224-225 (1996)

5. Zhou, J-R, Srivastava, HM, Wang, ZG: Asymptotic distributions of the zeros of a family of hypergeometric polynomials. Proc. Am. Math. Soc. 140, 2333-2346 (2012)

6. Ricci, PE: Generalized Lucas polynomials and Fibonacci polynomials. Riv. Mat. Univ. Parma 4, 137-146 (1995)

7. Amdeberhan, T: A note on Fibonacci-type polynomials. Integers 10, 13-18 (2010) 
10.1186/1029-242X-2013-466

Cite this article as: Halıc and Akyüz: A note on the roots of some special type polynomials. Journal of Inequalities and Applications 2013, 2013:466

Submit your manuscript to a SpringerOpen ${ }^{\circ}$ journal and benefit from:

- Convenient online submission

- Rigorous peer review

- Immediate publication on acceptance

Open access: articles freely available online

- High visibility within the field

- Retaining the copyright to your article

Submit your next manuscript at $\boldsymbol{s p r i n g e r o p e n . c o m ~}$ 